\title{
Residual thermic effects of diet induced thermogenesis (RDIT) in aging lean and obese LA/Ntul//-cp (corpulent) rats
}

\begin{abstract}
Diet induced thermogenesis has been proposed to account for up to $15 \%$ of the caloric content of an ingested meal and to become decreased with aging and in obesity. Genetically obese rodents have been shown to exhibit impairments in the thermic responses to diet and environment, which may partially account for an improved caloric efficiency and to contribute to their increased propensity to become obese. In the present study we sought to determine the thermic responses to diet and environment in aging obese rats when young, middle aged, and aged. Resting oxygen consumption tended to decrease with advancing age and the thermic responses were lower than predicted in obese than in lean rats. This study provides important new insights regarding the thermogenic effects of diet and diet induced thermogenesis and their potential contributions to mechanisms of energy balance across the spectrum of aging in lean and obese LA/Ntul//-cp rats.
\end{abstract}

Keywords: thermogenesis, aging, obesity, mammalian species
Volume II Issue 4 - 202 I

\author{
Orien L Tulp,' Susan P DeBolt,' Aftab R \\ Awan, ${ }^{2}$ George P Einstein ${ }^{1-3}$ \\ 'College of Medicine, University of Science Arts and Technology, \\ Montserrat, BWI \\ ${ }^{2}$ University of Health and Humanities, Virgin Islands, BWI \\ ${ }^{3}$ Department of Experimental Medicine, Einstein Medical \\ Institute, USA
}

\begin{abstract}
Correspondence: Orien L Tulp, Colleges of Medicine and Graduate Studies, University of Science Arts and Technology, Montserrat, BVI; University of Health and Humanities, Virgin Islands, and Dept of Experimental Medicine, Einstein Medical Institute, North Palm Beach, FL, USA, Email o.tulp@usat.edu
\end{abstract}

Received: August II, 202I | Published: August 23, 202 I

\section{Overview}

The phenomena of Diet-Induced Thermogenesis (DIT) represents the combined metabolic contributors to heat generation following ingestion of meals in mammalian species, their net overall thermic impact on processes of digestion, and post-prandial nutrient and substrate assimilation. Impairments in DIT have often been implicated in the caloric efficiency of excess weight gain and the predisposition for adiposity in man and animals, where it may account for up to $15 \%$ of the caloric content of a meal becoming dissipated as heat rather than being stored as fat. To determine the residual effects of DIT [RDIT] on energy expenditure in aging and obesity, measures of post-prandial residual RMR and the thermic responses to diet were determined in lean and obese rats of the LA/N//-cp strain over much of the course of the lifespan of the animals. Groups of congenic female lean and obese LA/N//-cp rats from 3 to 28 months of age were fed Purina stock diets or the stock diet plus a thermogenic 16\% sucrose supplement added to their drinking water. Rats were grouped by littermate pairs into three broad age categories with mean ages of 4,14 , and 24 months. Measures of total caloric intake relative to controls were determined over 24-hour periods and showed that caloric intake remained similar in lean rats throughout the age spectrum observed, but caloric intake was greater in the obese phenotype at all ages measured but was not materially impacted by the sucrose supplement. Rats were subjected to measures of resting and residual thermic effects of diet induced thermogenesis (VO2) following feeding and supplementation where the $16 \%$ sucrose solution supplement was added to their drinking water and normal diet. Resting VO2 of lean rats were greater than in obese rats in the fasting, fed, and sucrose supplemented groups at all ages studied, and remained at similar levels throughout the age span in the lean phenotype. The 4 hour post prandial residual thermic effects of Purina-fed, plus sucrose, or both nutriments after 4 hours post feeding were minimal in magnitude in both phenotypes and were not impacted with older age at 14 and 24 months of age in the post prandial state. These results indicate that the physiologic phenomena of the residual thermic effects of DIT are modest in magnitude, consistent with an age associated impairments in cold and non-shivering thermogenesis reported elsewhere in the obese phenotype of this strain. The observations reported are consistent with earlier observations in younger animals of this strain and may be a contributing factor in the development and expression of obesity via epigenetic modulation of alterations in energy metabolism pathways and in parameters of caloric efficiency in response to factors of diet, environment and aging.

\section{Introduction}

The phenomena of diet induced thermogenesis (DIT) includes neuroendocrine and metabolic elements in addition to the physiologic energy expended in the luminal digestive processes of meal digestion. ${ }^{1}$ The processes include ATP expenditure that is directly linked to the Musculo intestinal digestive processes, active transport of nutrients, micronutrients and substrates derived from the digesta and their related thermic activities including energy dependent nutrient absorption and metabolism. Up to $15 \%$ of the calories consumed may be lost in the immediate 3 to 4 hours of post prandial metabolism following meal ingestion via the cumulative physiologic and neuroendocrine effects of DIT. ${ }^{1,2}$ A number of authors have proposed that potential defects or impairments in DIT may be a measurable contributor to the efficiency of caloric utilization and energy storage. ${ }^{3,4}$ Obesity occurs in the congenic LA/N//-cp rat strain as the consequence of the autosomal recessive (-cp) trait and appears in approximately $25 \%$ of the offspring of breeding pairs that are heterozygous for the Koletsky ${ }^{5}$ $(-c p)$ trait by 5 to 6 weeks of age as determined by measurable differences in body weight and other physical characteristics of early development of obese stigmata. ${ }^{5-7}$ The lean phenotype of this strain retains the physiologically normal characteristics of the LA/N background, while the obese phenotype develops early onset of adiposity leading to overt obesity by early adulthood, accompanied with mild to moderate carbohydrate intolerance, hyperlipidemia, 
hyperamylinemia, and alterations in endocrine functions that are often characteristic accompaniments of obesity. ${ }^{6-8}$ The obese phenotype remains normotensive and non-diabetic throughout their lifespan despite the magnitude of obesity exhibited, and to date, the obese phenotype has failed to produce offspring, thus the need to breed animals that are heterozygous for the $(-c p)$ trait to generate offspring expressing the obese phenotype. ${ }^{6,9}$

The LA/N//-cp strain was originally developed by Hansen by breeding the $(-c p)$ trait from the Koletsky rat into the LA/N (ListerAlbany/NIH) followed by 12-18 cycles of backcrossing to select for the virtual homogeneity of the $(-c p)$ trait transmission to form a new strain. ${ }^{6,10,11}$ The new strain resulted in a new animal model for studies of obesity and diabetes research with a model expressing otherwise physiologically normal metabolic characteristics, such that the independent effect of the obese phenotype could be investigated without the confounding effects of secondary metabolic contributors. The LA/N background strain was noted for its relatively long life-span under standard laboratory conditions, and lean LA/Ntul//-cp rats have been reported to survive for up to 4 years and their obese littermates up to 3 years under conventional laboratory conditions.

The LA/N-cp strain relocated to the Drexel colony was further designated as the LA/tul//-cp sub-strain by the NIH to distinguish it from other related stains bearing the $(-c p)$ trait. ${ }^{11-13}$ Diet Induced Thermogenesis [DIT] represents the increases in energy expenditure following meal consumption, and typically represents the combined thermic effects of digestive activity, activation of heat generation in brown adipose tissue (BAT) and other tissues including liver and muscle following meals. ${ }^{1-4}$ Few studies have reported the duration or residual impact of the thermic effects of feeding, and the residual thermic impacts while assumed to remain contributory to energy balance have not previously been reported in the corpulent rat strains. The obese phenotype of the LA/N//-cp rat has been reported to demonstrate an improvement in caloric efficiency following normal feeding patterns $\mathrm{s}^{4,14}$ presumed a consequence of impaired neuroendocrine and endocrinologic responses to diet, and where macronutrient distribution likely plays a major role in the activation and expression of DIT. ${ }^{15,16}$ The effects of aging on thermogenesis are similarly unclear, as few rodent animal models demonstrate sufficient longevity to fully investigate the contributory metabolic mechanisms in their entirety. The LA/N//-cp model, developed from the longevityprone $\mathrm{LA} / \mathrm{N}$ background, combined with its congenic and specific pathogen free (SPF) status offers such a model, as the independent variables of aging may be observed where the only variation between the lean and obese phenotypes is the expression of the corpulent (-cp) trait, as originally derived from the Koletsky rat by Hansen.

The uncoupling of oxidative phosphorylation in brown adipose tissue (BAT) has been attributed to be a primary element of the phenomena of diet induced thermogenesis in man and animals ${ }^{15,16}$ where it has been shown to increase in thermogenic activity in response to carbohydrate feeding and adrenergic stimulation. ${ }^{7,17}$ In contrast to white adipose tissue, BAT tissues contain abundant specialized mitochondria capable of generating heat directly upon sympathetic innervated via $\beta 3$ adrenergic receptor activation. ${ }^{7,17-19}$ In animal studies, BAT thermogenesis in $(-c p)$ strains has been shown to become impaired due to a combination of neuroendocrine dysfunction and insulin insensitivity. ${ }^{18,19}$ The thermic responses in BAT have been demonstrated to be the consequence of uncoupling of oxidative phosphorylation, resulting in heat generation due to the breaking of the high energy phosphate bonds of ATP to ADP with the liberation of energy from the high energy phosphate bond in the form of heat. ${ }^{17}$ In animal studies, interscapular BAT (IBAT) mass has been shown to increase via hyperplasia in early life of the rat in response to both dietary and environmental factors, thereby enabling previously exposed animals to have a potentially greater thermogenic capacity to buffer against increases in cold exposure. ${ }^{4,7,20}$ Early overnutrition or adrenergic stimulation increases IBAT mass and cellularity compared to normally fed and reared lean littermates, and which thermogenic potential may continue generating thermogenic activity well into adulthood, since the BAT adipocytes, once formed in early life, remain present thereafter. ${ }^{21}$ The thermic responses of early cafeteria fed rats have been shown to be due to a combination of sympathetic and thyroidal influences. ${ }^{22,23}$ Other substrates capable of stimulating thermogenesis in BAT and other issues include glucose and fatty acids. ${ }^{15,16}$ The energy wasting hepatic $\alpha$-glycerophosphate shuttle correlates with circulating T3 availability, in contrast to the complimentary malateaspartate shuttle which favors energy conservation in fatty and other tissues..$^{24,25}$ The chronic hyperinsulinemia of the obese phenotype also favors energy conservation and net caloric efficiency in part likely due to insulin inhibition of BAT and other biochemical mechanisms generating thermic activity.

Glucose infusion has also been linked to transient thermic activity by an immediate increase in $\mathrm{VO} 2$ due to peripheral uptake and oxidation in liver and other issues. ${ }^{17,26}$ Glucose is absorbed rapidly following both oral and intravenous administration, where it can undergo cellular uptake without intestinal digestion and in peripheral tissues via a number of Glut receptors, some of which including Glut4 require insulin. ${ }^{27}$ BAT thermogenesis is also increased in response to glucose availability, independent of the GDP stimulatory pathway but may be impaired in insulin resistant states..$^{18,19}$

\section{Materials and methods}

The animals used in this study were obtained from the Drexel University Breeding Colony maintained by Drs O. Tulp and S. Dubin and maintained as littermate pairs in a dedicated animal room under standard conditions of light, temperature and relative humidity as described previously. ${ }^{4,7,28,29}$ All animals were generated from the same breeding pairs of LA/Ntul//-cp rats proven to be heterozygous for the $(-c p)$ trait via evidence of obese offspring among previous test litters. In this strain, the $(-c p)$ trait is inherited as an autosomal recessive trait, resulting in two phenotypes (lean and obese) and yielding three inheritance patters: homozygous lean (-/-), heterozygous lean (-/-cp) and homozygous obese $(-\mathrm{cp} / \mathrm{-cp})$. Obese offspring were identified in littermate pairs by gender (one lean, one obese, both of the same gender) by 5 to 6 weeks of age based on their physical demonstration of obese stigmata. Animals remained in the study groups from 3 months to 28 months of age and were divided into three mean age groups: with mean ages of 4 (range 3 to 6), 14(range 10-20), and 24 (range 2028) months of age. Specific pathogen free (SPF) status was confirmed by periodic necropsy of representative animals to confirm the absence of infectious illness or disease. The conditions of temperature and humidity with a reverse light cycle throughout the study have been described elsewhere. ${ }^{4,7,12}$ Animals were routinely housed in plexiglass cages lined with pine shavings, 2 to 4 animals per cage depending on size, to ensure adequate ambulatory and rearing space and based on AVMA and standard animal care requirements and maintained in the same animal room isolated from other animals throughout their life span. Animal care was maintained by the same animal care staff and handlers throughout the study to minimize the impact of potential undue stress on analytical measurements. This study was approved by the Animal Care and Use Committees of Drexel University and USAT/UHHVI. 
Measures of cumulative caloric intake were obtained as discussed previously. ${ }^{4,28}$ Briefly animals were placed in individual metabolic cages and allowed to acclimatize to the enclosure prior to the collection of solid food and liquid intake data over a 2 to 3 day repeat 24-hour durations. Grams of food and fluid intake, corrected for spillage was recorded to the nearest gram and multiplied by the caloric density of the solid or liquid diet (Purina \# 5012, stated as $3.4 \mathrm{kcals} / 14.2$ joules/ gram on the certificate of analysis). The nutritional composition of the Purina diet contained $55.6 \% \mathrm{CHO}, 22.5 \%$ protein, $4.5 \%$ fats, $4.6 \%$ crude fiber, $6 \%$ ash and $1.2 \%$ essential vitamins and minerals, and reported an energy density of $3.4 \mathrm{kcal} / \mathrm{gram}$ ( 14.2 joules/gram) based on the manufacturer's certificate of analysis. ${ }^{4,7}$ The sucrose water was prepared daily with 16 grams of sucrose with added house water q.s to $100 \mathrm{ml}$ volume of solution and a computed caloric density of 64 $\mathrm{kcal} / 100 \mathrm{ml}$. for the sucrose solution and was corrected for excess drippage.

All measures of VO2 were taken during the light phase of the lightdark cycle after a brief (10 minute) period of equilibration in a Collins small animal respirometry apparatus as modified in our laboratory to accommodate a closed circuit system. ${ }^{7}$ Animals were deemed Specific Pathogen Free (SPF) by virtue of their isolated environment, personal protective equipment and attire (PPE) requirements for all attending staff and periodic necropsy analysis for evidence of confirmed negative status for infectious diseases common to rodents and other small animal species. Rats were fed a standard Purina stock diet \# 5412 and house water ad libitum or the Purina diet regimen plus a $16 \%$ sucrose solution in place of their drinking water for 3 to 4 weeks prior to obtaining measures of respiration.

The experimental design of this study was a conventional $2 \times 3 \times$ 4 design, with 2 phenotypes (lean and obese), 3 age groups (age range 3 to 28 months; mean age groups 4,14 , and 24 months of age) 4 total study parameters (VO2 at: a) fasting, b) after feeding Purina diet, c) fasting after sucrose, and d) 4 hours after feeding the sucrose liquid supplement). Measures of VO2 were obtained in a Collins Small Animal Respirometer closed circuit apparatus after $\mathrm{CO}_{2}$ removal via a $\mathrm{CO}_{2}$ absorbent. The apparatus was fitted with a one cubic foot volume plexiglass cannister in a closed-circuit system and the measures of oxygen utilization recorded from individual quietly resting animals with a spirometer apparatus (Warren Collins Co, Braintree, MA). All measures of $\mathrm{VO} 2$ were taken at $29^{\circ} \mathrm{C}$ deemed thermal neutrality for the rat as reported elsewhere ${ }^{7}$ by immersion of the sealed cannister in a closed-circuit system, maintained in a constant temperature circulating water immersion bath throughout the course of the measurements over a period of 30 to 45 minutes approximately 4 to 6 hours after the end of the dark cycle, when rats have been observed to undergo the most active phase of their feeding activity and when it was possible to determine the residual thermic effects of recent post prandial feeding activity. Food and fluid were provided rats ad libitum prior to placement in the cannister, but no food or liquid was provided the animals while resting comfortably in the $\mathrm{VO} 2$ cannister. Measures of barometric pressure and altitude of the laboratory were factored into the equation and attempts to keep all environmental conditions constant were rigidly adhered to throughout the study. Measures of $\mathrm{VO} 2$ reflective of residual post prandial DIT activity were reported a $\mathrm{ML} \mathrm{O}_{2} / \mathrm{kg}$ body weight ${ }^{-0.75}$ to allow for differences in body size and mass as described by Klieber and Wang et al. ${ }^{30,31}$ Weights of animals were recorded to the nearest gram with an Ohaus beam balance (Fisher Scientific) immediately prior to placement of the animal in the VO2 cannister. ${ }^{28}$ The determination of the Residual Thermic effect of Diet induced thermogenesis (DIT) was determined 4 hours after the last availability of food and was coined the term RDIT. House water was available ad libitum until placement of the animal in the thermogenesis apparatus.

\section{Results}

Cumulative mean daily caloric intake over 24-hour measurements of normally Purina-fed and supplemented rats is depicted in Figure 1, relative to lean control rats placed at $100 \%$ dietary consumption at 4 months of age. Lean and obese control data are depicted by the open bars, and the dietary treatments depicted in the solid bars. The data for the three lean age groups are depicted in the left panel of each figure, and the three obese phenotype groups by age in the right panel of the respective figures. Daily net caloric intake by lean rats remained similar at all ages studied and was unaffected by addition of the sucrose supplement to their drinking water, suggesting a normalization of appetite regulation mechanisms over the age spectrum studied in the lean phenotype. In contrast, absolute daily caloric intake in obese phenotype was increased by 40 to 100 percent or more at each age studied, and the hyperphagia of the obese phenotype is consistent with the greater size and mass of the obese animals at all ages. The addition of the sucrose supplement was also associated with a progressively greater daily caloric intake in the obese phenotype compared to normally fed lean animals, but statistically significant differences in sucrose stimulated caloric intake were modest $(\mathrm{p}=0.10)$, with a trend toward secondary appetite moderation.

\section{CALORIC INTAKE OF FED LEAN AND OBESE RATS}

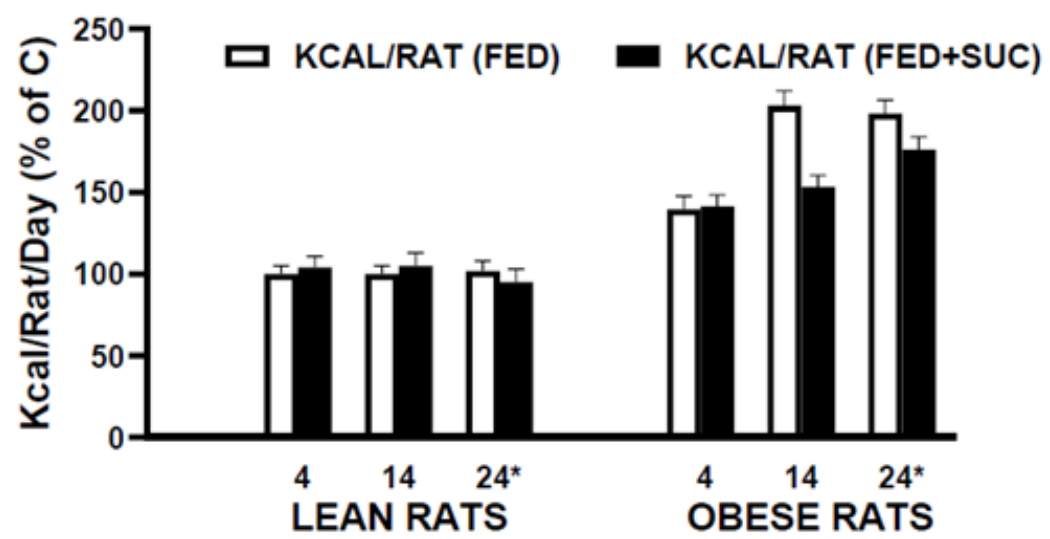

Figure I Daily caloric intake of rats expressed as a percent of lean control, where lean control at 4 months of age $=100 \%$. Data are mean $+/-$ I SEM, $n=4-8$ rats/age group per phenotype. $\mathrm{P}=<0.05$ lean vs obese at each age studied. Lean Sucrose vs control $=$ N.S. at all ages. $\mathrm{P}=\mathrm{N}$.S. obese control vs sucrose at ages 4 and 24 months, $\mathrm{p}^{+<0.05}$ for obese control vs. Sucrose at 14 months. 
The results of the fasting resting metabolic rates are depicted in Figure 2 and show that the post prandial RMR of lean animals remained similar with only a modest but non-significant decreasing trend $\{p=>0.05)$ throughout the spectrum of lean age groups studied.
In contrast, the RMR of obese rats was consistently lower than that recorded in the lean phenotype and progressively decreased significantly further in magnitude with each advancing age group in comparison to animals demonstrating the lean phenotype.

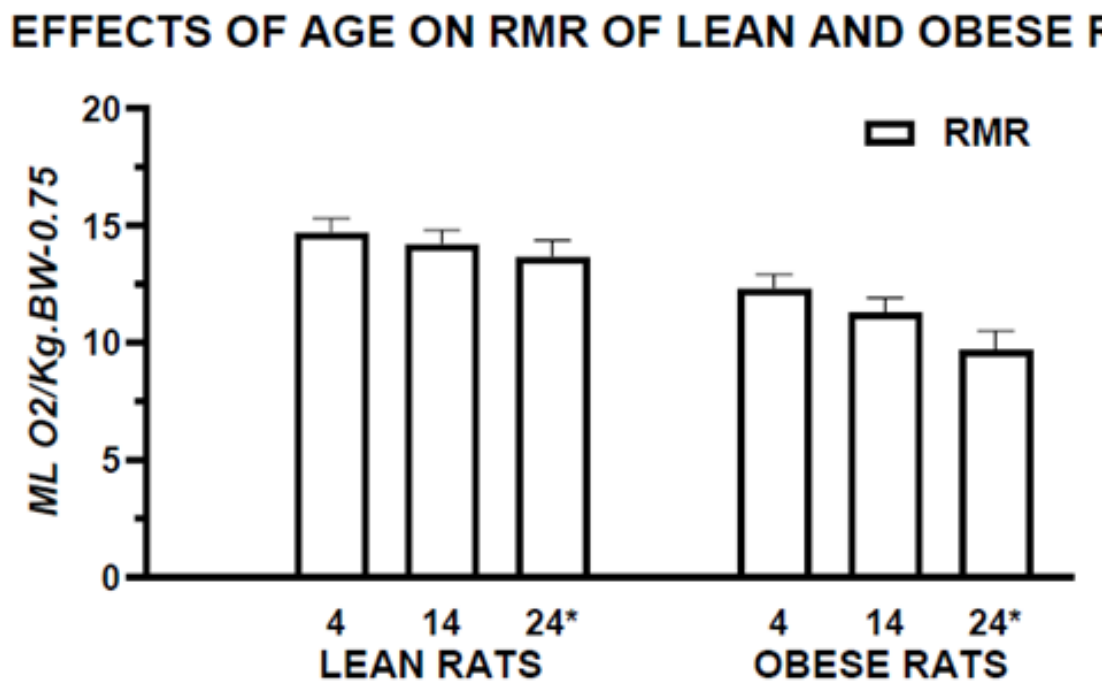

Figure 2 Effects of age group on RMR of lean and obese rats aged 4, I4, or 24 months of age. Data are mean $+/-$ I SEM, $n=4$ to 8 rats/group. *=incomplete data group, $n=4 . P=<0.05$ lean vs obese at ages 4,14 and 24 months. Effect of age were N.S. in lean rats, Effects of age were $<0.10$ in obese rats.

Measures of $\mathrm{VO} 2$ in animals offered the sucrose supplement pre-prandial are depicted in Figure 3 and indicate that RMRs were overwhelmingly similar to those depicted in Figure 2 above, and that post prandial measures of $\mathrm{VO} 2$ in the sucrose-fed lean animals were also similar to those of normally fed animals at all ages studied. In the obese phenotype, measures of RMR were similar to those depicted for fasting obese animals of each age group. The differences in VO2 between fasting RMR and sucrose supplemented group were not significant. Measures of VO2 in the chow fed group depicted in
Figure 4 were also greater than controls observed only in the 4-monthold lean animals, and the post prandial measures were not different from RMRs in lean animals thereafter or in obese animals at any age group. When the combined effects of sucrose and fed state were added as depicted in Figure 5, there was a small but non-significant trend toward an increase in VO2 in the lean group at each age studied, while no additive effects of feeding on $\mathrm{VO} 2$ were bserved in the obese phenotype at any age studied (Figure 5). ${ }^{15,21}$

\section{EFFECTS OF AGE AND SUCROSE ON VO2 IN LEAN AND OBESE RATS}

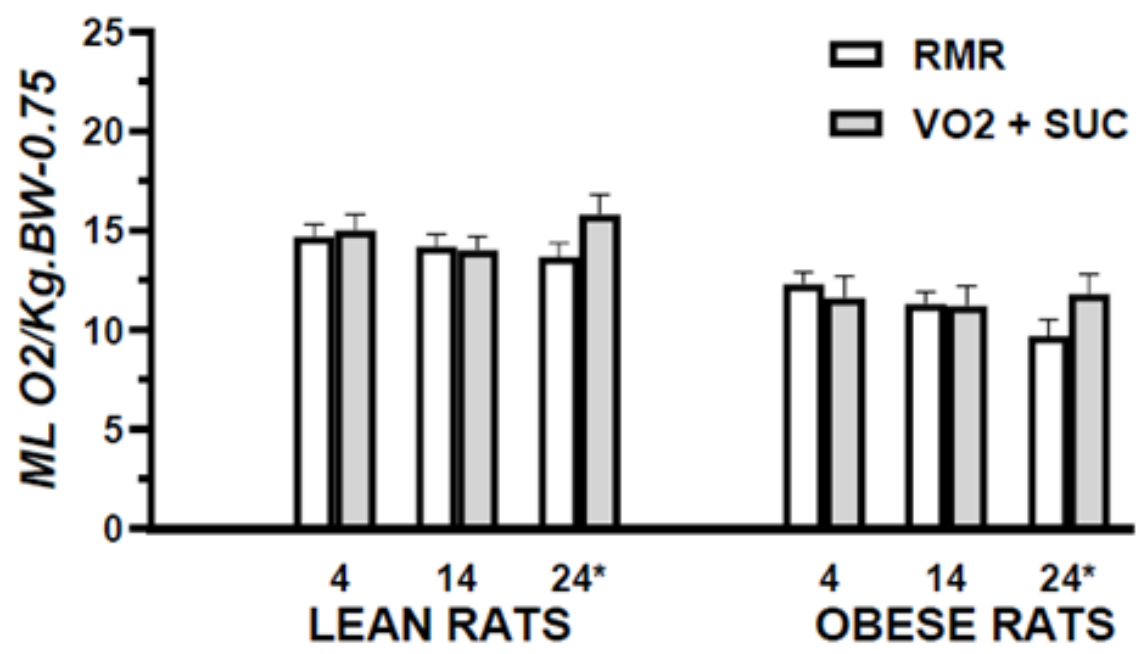

Figure 3 Effects of Age, phenotype and Sucrose on VO2in lean and obese rats. *=incomplete data set. 


\section{EFFECTS OF AGE AND FED STATE ON VO2 IN LEAN AND OBESE RATS}

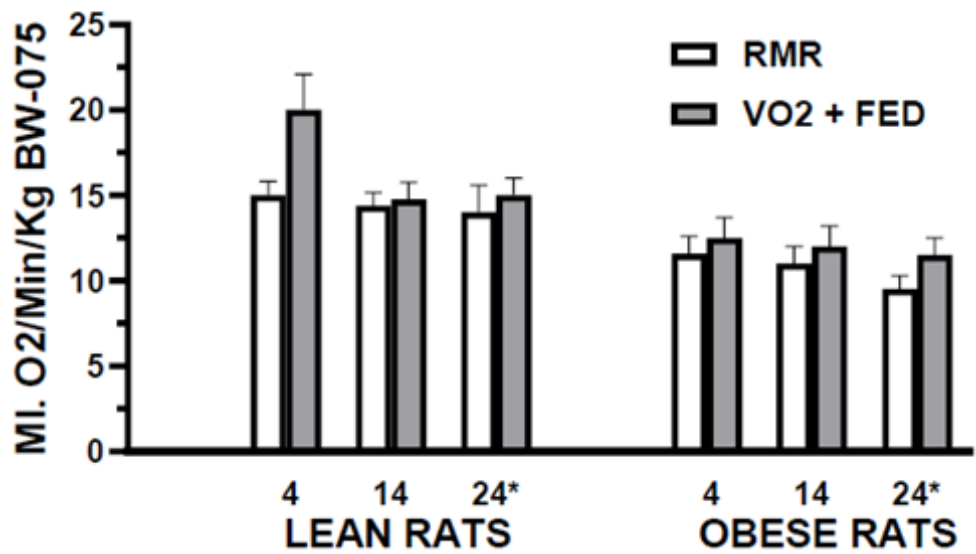

Figure 4 Effects of age and phenotype on VO2 of fed rats. Data are mean +/- I SEM, N=4-8 Rats/Group.

\section{EFFECT OF AGE, FED STATE AND SUCROSE ON VO2 OF LEAN AND OBESE RATS}

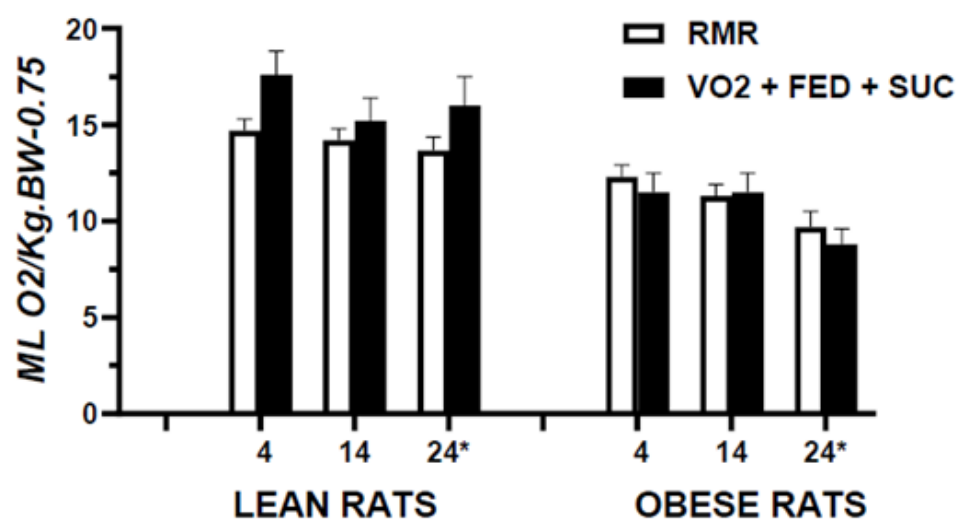

Figure 5 Effects of age, phenotype and diet (Fed+Sucrose) on VO2 of rats. Data are mean +/- I SEM, N=4-8 Rats/Group.

\section{Discussion}

Brown adipose tissue (BAT) has long been known to play a role in heat generation and temperature regulation in response to cold and dietary factors in man and animals. The topic of Diet-Induced thermogenesis or non-shivering thermogenesis as expressed in brown adipose tissue and its potential contributions to overall energy balance and long-term weight control however have been vigorously discussed for about the past 4 decades but without complete resolution. ${ }^{32-34}$ BAT has rich sympathetic innervation, via atypical $\beta 3$ adrenergic receptors, but continues to function following sympathetic denervation. ${ }^{35,36}$ Chemical blockade via a-methylparatyrosine or $\beta$ - blocking agents paints a different picture however, in that only about half of the DIT induced increases in thermic activity remain after chemical blockade, and the lipid locules diameters of brown adipocytes becomes increased, presumably due to impaired SNS activation. ${ }^{22,23,37}$ Early overnutrition via cafeteria feeding resulted in more than 2 -fold increase in IBAT mass, a 3-fold increase in IBAT cellularity and enhanced response to norepinephrine stimulation while IBAT lipectomy resulted in decreased response to noradrenaline stimulated thermogenesis, and in greater fat accretion than in non-IBAT lipectomized cafeteriafed Sprague-Dawley rats. ${ }^{37}$ In genetically predisposed Zucker rats, obesity still developed in the obese phenotypes even in the absence of hyperphagia, signaling metabolic alterations in one or more biochemical aspects of energy metabolism which likely results in greater caloric efficiency in the presence of the fatty trait. Direct measures of BAT or IBAT mass, cellularity or activity were not determined in the present report, but IBAT mass were found to be only modestly increased in sucrose fed lean animals with increasing age, while in the obese phenotype, interscapular BAT (IBAT) mass was greater than in lean rats both in absolute terms and as a proportion of body weight but was not further effected by the sucrose supplement, consistent with the presence of thermogenically less active beige IBAT as recently described by Rahman et al (2021) and noted to be present in the IBAT of obese rat strains expressing the -cp trait. ${ }^{12,18,19}$

An increase in metabolic rate, measured as an increase in oxygen consumption, is readily demonstrated in both human subjects and animal models in the immediate minutes and hours following meal ingestion and is attributed to thermogenic functions in BAT and other thermogenict issues due to complimentary increases in sympathetic, thyroidal and metabolic activities plus the physiological and biochemical energy cost of increased gastrointestinal activity. ${ }^{2,17}$ Four decades ago Rothwell and Stock demonstrated increased thermogenic 
responses in cafeteria fed rats due at least in part to increased sympathetic activity ${ }^{32}$ and Tulp et al demonstrated in cafeteria fed animals that ablation of the sympathetic component via administration of $\alpha$-methylparatyrosine could only block about $50 \%$ of the thermic response, thereby establishing a role for non-sympathetically mediated (non-SNS) contributions to DIT. ${ }^{22,23}$ In addition, thyroidectomy was shown to reduce both resting and minimal oxygen consumption even after feeding a thermogenic diet further clarifying the additive roles of thyroidal and sympathetic contributions to the physiologic process of DIT in rats. ${ }^{37}$ The nutritional component or components responsible for the majority of the immediate DIT response are predominantly attributed to the carbohydrate and protein content of the meal, where simple carbohydrates such as glucose or sucrose are more contributory in the early phases of the thermic response. Consumption of protein and other more complex macronutrients undergo the digestive and assimilative processes more slowly and over a longer duration, thereby contributing to the latter half of the DIT response typically extending beyond the first hours post feeding. ${ }^{1}$

The chemical form of the macronutrients consumed, in addition to non-caloric constituents including vitamins, minerals and indigestible fibers also combine in an additive manner to the determine the amplitude and the net area under the thermic curve of the cumulative thermic response. ${ }^{9,29}$ Starches, for example composed almost entirely of $\alpha-1,4$ and 1,6 linked glucose residues, undergo absorption of glucose moieties after intestinal digestion via luminal $\alpha$-glucosidases rapidly, and virtually $100 \%$ gastrointestinal absorption occurs within 30 to 45 minutes post ingestion, and thus would be expected to elicit an early glucose-mediated thermic response. Simple carbohydrates like sucrose undergo rapid luminal digestion via intestinal $\alpha$ - and $\beta$-glucosidases and subsequent insulin independent absorption in a rapid and efficient manner. Glucose may be absorbed directly into the circulation from the upper small intestine also within minutes of ingestion with peak glycemic responses occurring during the first 30 to 45 minutes following an oral glucose challenge in lean animals, ${ }^{28,29}$ thereby generating systemic glucose for uptake and oxidation in both visceral and peripheral tissues. Because glucose uptake occurs rapidly in the intestine, liver and nervous tissues independently of the Glut4 receptor mechanism, ${ }^{27}$ the insulin resistance which is common to obese states enables circulating early post prandial systemic glucose concentrations to rise and contribute to non-BAT thermogenic contributors to VO2 and caloric efficiency., $4,7,34$

Previous studies have shown that the RMR and the thermic effect of noradrenaline were diminished in the obese vs. the lean phenotype of the corpulent rat. ${ }^{7}$ These observations are consistent with the findings of Marette et al., ${ }^{18}$ and Bukowiecki et al., ${ }^{18}$ that demonstrated that insulin resistance likely plays a decisive role in expression of BATmediated thermogenesis in the obese phenotype of the LA/Ntul//-cp corpulent rat, and thus could explain at least in part the thermogenic impairment in the obese phenotype of corpulent rat. ${ }^{18,19}$ The thermic responses to antecedent diet beyond 4 or more hours post feeding are less clear however but are likely more similar to those of fasting animals, regardless of the specific composition of the prior feeding. In the present study, resting VO2 in lean rats remained at similar levels in both traditionally fasting and 4 hours post prandial animals, thus reflecting minimal sympathetically mediated contributions throughout much of the projected lifespan of the animals and has now been coined the term RDIT or residual thermogenesis of DIT.

The post prandial impact on RMR in fasting animals was predicted to be of longer lasting influence than that observed in the chow-fed or the sucrose fed group, due to the longer duration of luminal digestive actions than would be required for the more rapid digestion and absorption of sucrose to its monosaccharide elements and their subsequent gastrointestinal absorption, in contrast to the more complex macronutrient and micronutrient nature of the Purina chow diet. The duration of time since the last feeding in the current study was such that animals likely had returned to a near fasting state when the measures of $\mathrm{VO} 2$ were obtained however, thus minimizing the residual thermic responses to the antecedent dietary intake of the animal. The data shows that the effects of feeding both the Purina diet or the sucrose supplement, alone or in combination, had little impact on RDIT in the lean phenotype throughout the age spectrum studied, consistent with the virtual absence of age-linked changes in caloric efficiency reported previously. ${ }^{4}$ Had the measures of VO2 been made in yet older animals, it is likely that the age-associated deceasing tread in RMR apparent in the lean phenotype of the present study would have reached significance, as they did in the obese phenotype, and as they did in a related study. ${ }^{7}$ Thus RDIT, the post prandial residual thermic effects of DIT with aging were modest under the conditions examined in the present study in both lean and obese animals of this group of LA/Ntul//- $c p$ (Corpulent) rats. This study provides important new insights and pathways to understand and investigate thermogenic effects of diet and diet induced thermogenesis and their potential contributions to mechanisms of energy balance.

\section{Acknowledgments}

The authors wish to thank Dr. Stephen Dubin, Mr. Huang Peisong and Mary Victor of Drexel University for animal care and animal husbandry during this study.

\section{Conflicts of interest}

No conflict of interest was reported by the authors.

\section{Funding}

Institutional resources of USAT Montserrat, BWI, UHHVI, BVI, and the Einstein Medical Institute, and Drexel University.

\section{References}

1. Ho Ken KY. Diet-Induced thermogenesis:fake friend or foe? J Endocrinol. 2018;238(3):R185-R191.

2. Danforth E Jr. Diet and Obesity. Am J Clin Nutr. 1985;41(5 Suppl):1132-1145.

3. Tulp, OL, Awan, Aftab R, et al. Enhanced Caloric Efficiency Contributes to Adiposity in LA/N//-cp Corpulent Rats. Experimental Biology. 2021;35:S1.

4. Orien L Tulp. Effects of Aging, phenotype and carbohydrate feeding on caloric efficiency in the LA/Ntul//-cp (corpulent) rat. Adv Obes Weight Manag Control. 2021:11(1):5-11.

5. Koletsky S. Pathologic findings and laboratory data in a new strain of obese hypertensive rats. Am J Pathol. 1975;80 (1):129-142.

6. Greenhouse, DD. New Models of Genetically Obese Rats for Studies in Diabetes, Heart Disease, and Complications of Obesity. Ilar News. 1990;21(3):1-5.

7. Tulp, OL, Einstein, GP. Thermogenesis, aging and obesity in the LA/tul//cp (corpulent) rat. Adv Obes Weight Manag Control. 2021:11(2):37-43.

8. Huang HJ, Young AA, Koda JE, et al. Hyperamylinemia, hyperinsulinemia, and insulin resistance in genetically obese LA/N-cp rats. Hypertension. 1992;19. 
9. Michaelis OE IV, Ellwood KC, Tulp OL, et al. Effect of feeding sucrose or starch diets on parameters of glucose tolerance in the LA/N-corpulent rat. Nutr Res. 1986;6(2):95-99.

10. Hansen CT. The development of the SHR/N- and LA/N-cp (Corpulent) Congenic Rat Strains. In:New Models of Genetically Obese Rats for Studies in Diabetes, Heart Disease, and Complications of Obesity. NIH publication, Division of Research Services, Veterinary Resources Branch, National Institutes of Health, Bethesda, MD. 1988. p. 7-10.

11. Michaelis OE IV. In: New Models of Genetically Obese Rats for Studies in Diabetes, Heart Disease, and Complications of Obesity, Veterinary Resources Branch, Division of Research Services, NIH publication, Bethesda, MD. 1988. p. 13-15.

12. Tulp OL. Characteristics of thermogenesis, obesity, and longevity in the LA/N-cp rat. ILAR News. 1990;32(3):32-39.

13. EB Kahle, JM Dadgari, GA Dudley, et al. Adaptive Response of Enzymes of Carbohydrate and Lipid Metabolism to Exercise, in New Models of Genetically Obese Rats for Studies in Diabetes, Heart Disease, and Complications of Obesity. Veterinary Resources Branch, Division of Research Services, NIH publication, Bethesda, MD, 1988. p. 143-148.

14. Trayhurn P. Origins and early development of the concept that brown adipose tissue thermogenesis is linked to energy balance and obesity. Biochimie. 2017;134:62-70.

15. Zhao, JB Cannon, B Nedergaard J. Thermogenesis is beta3- but not beta1-adrenergically mediated in rat brown fat cells, even after cold acclimation. Am J Physiol. 1998;275(6):R2002-11.

16. Himms-Hagen J. Thermogenesis in BAT as an energy buffer. NEJM. 1984;311:1150-1156.

17. Marette A, Maurige P, Bukowiecki LJ, et al. Hormonal regulation of brown adipocyte glucose transport in genetic obesity. In:Obesity:Dietary factors and Control. In:DR Romsos, editors. Basel:Karger, 1991. p. 119130

18. Marette A, Tulp OL, Bukowiecki LJ. Mechanism linking insulin resistance to defective thermogenesis in brown adipose tissue of obese diabetic SHR/N -cp rats. Int J Obese. 1991;15:23-831.

19. Tulp OL. The development of brown adipose tissue during experimental over-nutrition in rats. Int J Obes. 1981;5(6):579-591.

20. Tulp OL, DeBolt SP. Aging and Obesity in the Corpulent Rat, in:Nestle Nutrition Series, Research and Practice in MNA and Aging. 1999. p. $149-155$.

21. Wolpert SI, RM Bye, OL Tulp. Effect of a methylparatyrosine on thermogenesis before and after cafeteria feeding in rats. Fed Proc. $43(5) ; 1984$

22. Tulp OL. Effect of a-methylparatyrosine on thermogenesis in lean and corpulent rats. Clin Res. 1984;32:237A.
23. Tatleman HM, Tyzbir RS, Tulp OL. Effects of overfeeding on BAT and liver mitochondrial metabolism and shuttle activity in adult rats. Fed Proc. 1981;40:871:3643

24. Galton DA, Bray G. Metabolism of $\alpha$-Glycerol Phosphate in Human Adipose Tissue in Obesity. The Journal of Clinical Endocrinology \& Metabolism. 1967;27(11):1573-1580.

25. Maliszewska, K, Kretowski A. Brown Adipose Tissue and Its Role in Insulin and Glucose Homeostasis. Int J Mol Sci. 2021;22(4):1530.

26. Mayes PA, Bender DA. Gluconeogenesis and control of the blood glucose Ch 19, In: Harper's Illustrated Biochemistry. 2003;19:160.

27. Vedula, U, Schnitzer-Polokoff R, Tulp OL. The effect of acarbose on the food intake, weight gain, and adiposity of $\mathrm{LA} / \mathrm{N}-\mathrm{cp}$ rats. Comp. Biochem. Physiol. A Comp Physiol. 1991;100(2):477-482.

28. Tulp, O.L. and Brown T. Effect of a Fructan-Chromium Complex on Glycemic Responses of Congenic Obese LA/Ntul/ / $c p$ Rats. J Nutr Health Food Eng. 2016;5(2):594-598.

29. Kleiber M. The Fire of Life:An Introduction to Animal Energetics. Wiley publishers, New York NY USA; 1961.

30. Wang, ZM, Zhang, J, Ying, Z, et al. Organ-Tissue Level Model of Resting Energy Expenditure Across Mammals: New Insights into Kleiber's Law. Int Scholarly Research Network. IBSN Zoology. 2012:Art ID 673050.

31. Rothwell NJ, Stock MJ. A role for brown adipose tissue in diet-induced thermogenesis. Nutrition \& Metabolism. 1997;5(6):650-656.

32. Labros Sidossis L, Shingo Kajimura S. Brown and beige fat in humans: thermogenic adipocytes that control energy and glucose homeostasis. $J$ Clin Invest. 2015;125(2):478.

33. Levin BE, Triscari J, Sullivan AC. Metabolic features of diet-induced obesity without hyperphagia in young rats. Amer J Physiol. 1986;251(3 Pt 2):R433-440.

34. Cox JE, Lorden JF. Dietary obesity:brown fat denervation fails to alter development or recovery. Am J Phsyiol. 1986;250(6 Pt 2):R1108-1116.

35. Tulp OL, D Root, R Frink. The effect of anti-hypertensive drug treatment on brown adipocyte diameter and locule distribution in rats. Comp Biochem Physiol. 1984;79(2):317-20.

36. Rahman Michael S, Einstein George P, Tulp Orien L. Autonomic Immunological and Endocrine influences on adipose tissue as an organ. Adv Obes Weight Manag Control. 2021:11(2):48-58.

37. Tulp OL, Frink R, Danforth E Jr. Effect of Cafeteria Feeding on Brown and White Adipose Tissue Cellularity, Thermogenesis, and Body Composition in Rats. $J$ Nutr. 112(12):2250-2260. 